\title{
The Impact of COVID-19 on Emergent Large-Vessel Occlusion: Delayed Presentation Confirmed by ASPECTS
}

(DD.J. Altschul, (D) N. Haranhalli, (D) C. Esenwa, (D) S.R. Unda, (D) R. de La Garza Ramos, (D). Dardick, (D). Fernandez-Torres, (D) A. Toma, (D) D. Labovitz, (D) N. Cheng, (D) S.K. Lee, (D) A. Brook, and DR. Zampolin O-

\begin{abstract}
BACKGROUND AND PURPOSE: Our hypothesis is that the COVID-19 pandemic led to delayed presentations for patients with acute ischemic stroke. This study evaluates the impact of the coronavirus disease 2019 pandemic on presentation, treatment, and outcomes of patients with emergent large-vessel occlusion using data from a large health system in the Bronx, New York.
\end{abstract}

MATERIALS AND METHODS: We performed a retrospective cohort study of 2 cohorts of consecutive patients with emergent large-vessel occlusion admitted to 3 Montefiore Health System hospitals in the Bronx from January 1 to February 17, 2020, (prepandemic) and March 1 to April 17, 2020 (pandemic). We abstracted data from the electronic health records on presenting biomarker profiles, admission and postprocedural NIHSS scores, time of symptom onset, time of hospital presentation, time of start of the thrombectomy procedure, time of revascularization, presenting ASPECTS, TICI recanalization score, mRS, functional outcomes, and mortality.

RESULTS: Of 179 patients admitted with ischemic stroke during the study periods, 80 had emergent large-vessel occlusion, of whom 36 were in the pandemic group. Patients in the pandemic group were younger (66 versus 72 years, $P<.061)$ and had lower ASPECTS $(7$ versus $9, P<.001)$ and took longer to arrive at the hospital (361 versus 152 minutes, $P<.004)$ with no other major differences. There was a decreased rate of thrombolysis administration $(22 \%$ versus $43 \%, P<.049)$ and a decreased number of patients treated with mechanical thrombectomy (33\% versus $61 \%, P<.013$ ).

CONCLUSIONS: The pandemic led to delays in patients arriving at hospitals, leading to decreased patients eligible for treatment, while in-hospital evaluation and treatment times remain unchanged.

ABBREVIATIONS: COVID-19 = coronavirus disease 2019; ELVO = emergent large-vessel occlusion

$I^{\mathrm{n}}$

March 2020, New York City became the epicenter of the coronavirus disease 2019 (COVID-19) pandemic, which disrupted regional systems of health care. The pandemic changed when and how patients presented for emergent evaluation. A recent study found a nearly $40 \%$ decrease in stroke-related imaging nationwide during the COVID-19 pandemic. ${ }^{1}$ While delays in treatment of myocardial infarction systems of care have been reported, the effect of the COVID-19 pandemic on systems of emergent large-vessel occlusion (ELVO) stroke care is unreported. $^{2}$

Received July 2, 2020; accepted after revision July 27.

From the Departments of Neurosurgery (D.J.A., N.H., S.R.U., R.d.L.G.R., J.F.-T., A.T.), Neurology (C.E.), and Radiology (D.L., N.C., S.K.L., A.B., R.Z.), Montefiore Medical Center/Albert Einstein College of Medicine, Bronx, New York; and Albert Einstein College of Medicine (J.D.), Bronx, New York.

Please address correspondence to David J. Altschul, MD, 3316 Rochambeau Ave, Bronx, NY 10467; e-mail: daltschu@montefiore.org; @DavidAltschulMD

- Indicates open access to non-subscribers at www.ajnr.org

Indicates article with supplemental on-line table.

http://dx.doi.org/10.3174/ajnr.A6800
Mechanical thrombectomy has been proved safe and effective in treating ELVO, but its success in decreasing morbidity depends on the prehospital system as well as a streamlined in-house process of care. ${ }^{3}$ Our current stroke triage process involves hospital prenotification, bypass of emergency department evaluation, direct advancement to CT scan assessment, and treatment before angiosuite arrival.

The aim of this study was to evaluate the impact of the COVID-19 pandemic on ELVO systems of care, treatment, and outcomes. We describe all cases of ELVO treated in our institution (Montefiore Health System) during the COVID-19 pandemic and compare them with those treated before the pandemic. Our hypothesis is that the COVID-19 pandemic led to delays in patients presenting to hospitals. This delay may have led to worse outcomes during the pandemic.

\section{MATERIALS AND METHODS Study Design and Participants}

The data that support the findings of the study are available from the corresponding author on reasonable request. This retrosp- 
ective cohort study included 2 cohorts of patients admitted to 3 of the hospitals in our health care network, including our comprehensive stroke center, which accepts transfers for complex cases from 8 community hospitals, from January 1 to February 17, 2020, (prepandemic) and March 1 to April 17, 2020, (pandemic). All patients diagnosed with ELVO were included. ELVO was defined as acute focal neurologic deficits with an associated occlusion of the internal carotid artery, M1 segment of the middle cerebral artery, M2 segment of the middle cerebral artery, or the basilar artery with radiologic confirmation by CTA. March 1 was the date chosen because it was the day of the first confirmed documented case in New York City. We also included patients with occlusions of the anterior cerebral artery, posterior cerebral artery, or vertebral artery and labeled them as "other" large-vessel occlusion. COVID-19 was confirmed by positive detection of Severe Acute Respiratory Syndrome coronavirus 2 RNA using real-time polymerase chain reaction assay testing performed within the hospital system or documented at an outside system before transfer. All patients presenting to our hospital during the pandemic period were tested for COVID-19 using real-time polymerase chain reaction assay testing.

This study was approved by the hospital institutional review board. Written informed consent was waived.

\section{Case Ascertainment and Study Variables}

Cases were identified through neuroradiology logs or neurologic consultation for transfers. Data abstracted from the medical records included demographics, clinical variables, laboratory values, and neuroimaging findings (initial ASPECTS, stroke treatment time points, results as they pertain to mechanical thrombectomy, outcome variables [discharge mRS 0-2], in-hospital mortality). Fourteen patients with inpatient presentation were excluded from analysis.

\section{Statistical Analysis}

We compared baseline characteristics between prepandemic and pandemic groups, performing parametric and nonparametric analyses with the $t$ test, $\chi^{2}$ test, and Mann-Whitney $U$ test as appropriate. No imputation was made for missing data. Missing data are labeled in the footnotes of the On-line Table. Main outcomes, poor outcomes (discharge mRS 3-5), and in-hospital mortality) with a $\chi^{2}$ test of $P<.05$ were analyzed through univariate and multivariate logistic regression controlling for explanatory variables. Multivariate analysis was performed with factors with $P<.05$ on univariate analysis controlling for explanatory variables with $P<.25$ on univariate analysis.

\section{RESULTS}

We found a total of 80 patients with ELVO during the entire study period, with 44 treated before and 36 during the COVID-19 pandemic. There was an $18 \%$ decrease in overall large-vessel occlusion volume during the pandemic. Pandemic patients were younger than those prepandemic ( 66 versus $72, P<.061$ ); however, this difference was not significant. There was no significant difference in comorbidities or race or ethnicity between the 2 groups. In the pandemic cohort, $38.7 \%$ tested positive for COVID-19 at admission.
Compared with the prepandemic cohort, pandemic patients with ELVO presented with a lower ASPECTS score (7 versus 9, $P<.001$ ), despite no differences in occlusion location, and arrived at the hospital later (361 versus 152 minutes, $P<.012$ ). There was a significantly lower rate of thrombolysis administration (22\% pandemic versus $43 \%$ prepandemic, $P=.049$ ) and a reduced proportion of patients treated with mechanical thrombectomy (33\% versus $61 \%, P=.013$ ). There was no significant difference in poor functional outcomes as scored using the mRS grades 3-5 (88.2\% versus $69.6 \%, P=.08$ ). There was no significant difference in mortality for patients with ELVO in prepandemic versus pandemic cohorts ( $15 \%$ versus $28.1 \%, P=.173$ ) (On-line Table).

\section{DISCUSSION}

We aimed to evaluate the effect of the COVID-19 pandemic by comparing two 6-week epochs. We evaluated sociodemographic data, comorbidities, established treatment time parameters, established imaging parameters, neurologic outcomes, and mortality between the 2 groups.

Patient symptom-onset-to-hospital times more than doubled in the pandemic period, and patients presented with significantly lower ASPECTS scores. Because intravenous thrombolysis and mechanical thrombectomy have either strict time and/or neuroimaging criteria per current guidelines, delays to care have a cascading effect, as reflected in the significantly fewer pandemic patients undergoing these hyperacute stroke therapies. ${ }^{4}$ In our 2 cohorts, there was no significant difference in race, ethnicity, vascular risk factors, or comorbidities (On-line Table). There was no significant difference in where (direct to a comprehensive stroke center versus a primary stroke center) patients presented between the 2 groups.

The reasons for delays in seeking care are multifactorial. A New York statewide shelter-in-place order and widespread media coverage of overwhelmed emergency health system resources likely helped mitigate the spread of COVID-19 but may have also unintentionally led to underuse of the emergency health system for other emergencies. This finding was not unique to New York City because similar delays were seen in other areas. ${ }^{5-7}$ One possibility is that patients with less severe symptoms did not seek early care; however, we did not see a significant difference in presenting NIHSS scores between the 2 groups. Other studies used lastknown-well times to predict a delay of onset; this data point is often subject to miscalculation and bias. This study is the first to confirm objectively using ASPECTS that patients had a higher degree of completed infarct when presenting to the hospital during the pandemic. While delays to presentation could be the main reason for the objective difference in ASPECTS at presentation, another possibility is that the multisystem inflammatory syndrome and hypercoagulable state associated with COVID-19 create a more severe stroke syndrome at presentation. ${ }^{8}$

Our hospital continued to treat patients regardless of their COVID status on the basis of our prepandemic standards of care. This approach did not impact our in-hospital triage and treatment times. The mortality rates were higher in the pandemic cohort; however, they did not reach clinical significance. Similar smaller reviews have suggested poor outcomes for patients with stroke who arrived during the pandemic. ${ }^{9}$ Further multicenter studies are needed to confirm these findings. 
The limitations of this study are its retrospective and singlecenter design and the sample size being small for the patients in the pandemic group.

\section{CONCLUSIONS}

Acute stroke is an example of when the COVID-19 pandemic led to "collateral damage" for noninfected patients. Awareness of this impact is a crucial first step in mitigating unintended health consequences during a massive emergency response.

Disclosures: David J. Altschul—UNRELATED: Consultancy: Stryker/MicroVention; Grants/Grants Pending: National Institutes of Health, Comments: R01 Subaward. Allan Brook-RELATED: Consulting Fee or Honorarium: InNeuroCo.

\section{REFERENCES}

1. Kansagra AP, Goyal MS, Hamilton S, et al. Collateral effect of Covid19 on stroke evaluation in the United States. $N$ Engl J Med 2020;383:400-01 CrossRef Medline

2. Tam CF, Cheung KS, Lam S, et al. Impact of coronavirus disease 2019 (COVID-19) outbreak on ST-segment-elevation myocardial infarction care in Hong Kong, China. Circ Cardiovasc Qual Outcomes 2020;13: e006631 CrossRef Medline
3. McTaggart RA, Yaghi S, Cutting SM, et al. Association of a primary stroke center protocol for suspected stroke by large-vessel occlusion with efficiency of care and patient outcomes. JAMA Neurol 2017;74;793-800 CrossRef

4. Powers WJ, Rabinstein AA, Ackerson T, et al. Guidelines for the Early Management of Patients with Acute Ischemic Stroke: 2019 Update to the 2018 Guidelines for the Early Management of Acute Ischemic Stroke-A Guideline for Healthcare Professionals from the American Heart Association/American Stroke Association. Stroke 2019;50:e344-418 CrossRef Medline

5. Teo KC, Leung WC, Wong YK, et al. Delays in stroke onset to hospital arrival time during COVID-19. Stroke 2020;51:2228-31 CrossRef Medline

6. Schirmer CM, Ringer AJ, Arthur AS, et al. Delayed presentation of acute ischemic strokes during the COVID-19 crisis. J Neurointerv Surg 2020;12:639-42 CrossRef Medline

7. Qin C, Zhou L, Hu Z, et al. Clinical characteristics and outcomes of COVID-19 patients with a history of stroke in Wuhan, China. Stroke 2020;51:2219-23 CrossRef Medline

8. Yaghi S, Ishida K, Torres J, et al. SARS2-CoV-2 and stroke in a New York healthcare system. Stroke 2020;51:2002-11 CrossRef Medline

9. Wang A, Mandigo GK, Yim PD, et al. Stroke and mechanical thrombectomy in patients with COVID-19: technical observations and patient characteristics. J Neurointerv Surg 2020;12:648-53 CrossRef Medline 
On-line Table: Baseline characteristics and outcomes of patients with ELVO prepandemic and during the pandemic

\begin{tabular}{|c|c|c|c|c|}
\hline Variables & All $(n=80)$ & Prepandemic $(n=44)$ & Pandemic $(n=36)$ & $P$ Value \\
\hline Age (mean) (SD) (yr) & $69.75(14.41)$ & $72.48(14.96)$ & $66.42(13.15)$ & $.061^{\mathrm{a}}$ \\
\hline Male sex (No.) (\%) & $39(48.8)$ & $21(47.7)$ & $18(50)$ & .486 \\
\hline \multicolumn{5}{|l|}{ Race } \\
\hline White (No.) (\%) & $14(17.5)$ & $6(13.6)$ & $8(22.2)$ & .142 \\
\hline African American (No.) (\%) & $27(33.8)$ & $15(34.1)$ & $12(33.3)$ & \\
\hline Hispanic (No.) (\%) & $24(30)$ & $11(25)$ & $13(36.1)$ & \\
\hline Other (No.) (\%) & 15 (18.8) & $12(27.3)$ & $3(8.3)$ & \\
\hline \multicolumn{5}{|l|}{ Vascular risk factors } \\
\hline Hypertension (No.) (\%) & $62(77.5)$ & $35(79.5)$ & $27(75)$ & .628 \\
\hline Hyperlipidemia (No.) (\%) & $37(46.3)$ & $20(45.5)$ & $17(47.2)$ & .875 \\
\hline Diabetes (No.) (\%) & $31(38.8)$ & $17(38.6)$ & $14(38.9)$ & .982 \\
\hline Atrial fibrillation (No.) (\%) & $21(26.3)$ & $15(34.1)$ & $6(16.7)$ & .078 \\
\hline Congestive heart failure (No.) (\%) & $12(15)$ & $9(20.5)$ & $3(8.3)$ & .131 \\
\hline Smoking (No.) (\%) & $19(23.8)$ & $13(29.5)$ & $6(16.7)$ & .211 \\
\hline \multicolumn{5}{|l|}{ Clinical presentation ${ }^{\mathrm{b}}$} \\
\hline Direct admission & $53(66.3)$ & $29(66.7)$ & $24(66.7)$ & .929 \\
\hline Transfer from other hospital & $27(33.7)$ & $15(33.3)$ & $12(33.3)$ & \\
\hline mRS baseline (median) (IQR) & $1(0-3)$ & $2(0-3)$ & $0(0-1)$ & $.042^{\mathrm{a}}$ \\
\hline NIHSS on admission (median) (IQR) & $15(9-20)$ & $14(8-20)$ & $16(12-20)$ & .190 \\
\hline ASPECTS on admission (median) (IQR) & $8(6-9)$ & $9(7-10)$ & $7(4-8)$ & $<.001^{\mathrm{a}}$ \\
\hline \multicolumn{5}{|l|}{ Location of occlusion } \\
\hline Right MCA (No.) (\%) & $24(30)$ & $14(31.8)$ & $10(27.8)$ & .695 \\
\hline Left MCA (No.) (\%) & $27(33.8)$ & $12(27.3)$ & $15(41.7 .5)$ & .176 \\
\hline Right ICA (with carotid-T) (No.) (\%) & $4(5)$ & $2(4.5)$ & $2(5.6)$ & .837 \\
\hline Left ICA (with carotid-T) (No.) (\%) & $4(5)$ & $2(4.5)$ & $2(5.6)$ & .837 \\
\hline Right ICA (No.) (\%) & $7(8.8)$ & $3(6.8)$ & $4(11.1)$ & .499 \\
\hline Left ICA (No.) (\%) & $9(11.3)$ & $4(9.1)$ & $5(13.9)$ & .499 \\
\hline Basilar artery (No.) (\%) & $4(5)$ & $3(6.8)$ & $1(2.8)$ & .409 \\
\hline Other & $9(11.3)$ & $4(9.1)$ & $5(13.9)$ & .499 \\
\hline \multicolumn{5}{|l|}{ Treatment information } \\
\hline Thrombolysis IV (No.) (\%) & $27(33.8)$ & $19(43.2)$ & $8(22.2)$ & $.049^{\mathrm{a}}$ \\
\hline Thrombectomy (No.) (\%) & $39(48.8)$ & $27(61.4)$ & $12(33.3)$ & $.013^{\mathrm{a}}$ \\
\hline \multicolumn{5}{|l|}{ Time points ${ }^{c}$} \\
\hline \multicolumn{5}{|l|}{ All patients with acute stroke $(n=66 / 80)$} \\
\hline Onset to first hospital (median) (IQR) (min) & $217(105-575)$ & $152(74.5-470)$ & $361(161-1020)$ & $.012^{\mathrm{a}}$ \\
\hline Onset to our institution (median) (IQR) (min) & 331 (161-702) & $241(133-472)$ & $604(235-1160)$ & .181 \\
\hline$>6 \mathrm{~h}$ from onset to door (No.) (\%) & $31(47)$ & $13(35.1)$ & $18(62.1)$ & $.03^{\mathrm{a}}$ \\
\hline \multicolumn{5}{|l|}{ Patients undergoing thrombectomy $(n=39 / 80)$} \\
\hline Door to groin (median) (IQR) (min) & $82(52-124)$ & $80(44-130)$ & $84(68-110)$ & 1.00 \\
\hline Groin to recanalization (median) (IQR) (min) & $41(36-67)$ & $41(37-58)$ & $38.5(33-68)$ & .577 \\
\hline $\mathrm{TICl}$ score $\geq 2 \mathrm{~b}$ (No.) (\%) & $32(82.1)$ & $22(81.5)$ & $10(83.3)$ & .889 \\
\hline Day 1 NIHSS (median) (IQR) & $11(5-18)$ & $10(4.5-17)$ & $12(6-19)$ & .361 \\
\hline \multicolumn{5}{|l|}{ Outcomes d } \\
\hline Poor outcomes (mRS 3-5) (No.) (\%) & $46(80.7)$ & $30(88.2)$ & $16(69.6)$ & .08 \\
\hline In-hospital mortality (No.) (\%) & $15(20.8)$ & $6(15)$ & $9(28.1)$ & .173 \\
\hline
\end{tabular}

Note:-IQR indicates interquartile range.

${ }^{a}$ Significant $(P<.05)$.

${ }^{\mathrm{b}}$ Missing values for clinical presentation: NIHSS $2 / 80$ (2.5\%) and ASPECTS $5 / 80$ (6.3\%).

${ }^{C}$ Missing data for treatment information: onset to first hospital $6 / 66(9 \%)$, onset to our institution $1 / 66(1.5 \%)$, door to groin $1 / 39(2.6 \%)$, groin to recanalization $4 / 39$ (10.3\%), and day 1 NIHSS $8 / 80$ (10\%).

${ }^{d}$ Missing data for outcomes: discharge mRS 8/80 (10\%). 